\title{
INTERFACIAL ENGINEERING FOR OPTIMIZED ADHESION IN POLYMERIC COMPOSITE MATERIALS
}

\author{
LAWRENCE T. DRZAL
}

Department of Chemical Engineering, Composite Materials and Structures Center, Michigan State University, East Lansing, MI 48824-1326

\begin{abstract}
Fiber-matrix adhesion is a variable to be optimized so that optimum composite mechanical properties can be achieved in polymer matrix composites. The contemporary view of adhesion rests on an "interphase" model in which not only the actual chemical and physical interactions between fiber and matrix are considered but also the structure and properties of both the fiber and the matrix in the region near the interface. The optimum design methodology starts with the specification of the fiber and matrix from a structural consideration. Once the constituents are selected, the focus is on the creation of a beneficial fiber-matrix "interphase". This region where the fiber and matrix interact has to be designed for both "processing" and "performance". Although no quantitative algorithm is available for interphase optimization, various thermodynamic principles coupled with experimental data can be used to qualitatively design the optimum interphase. Examples will be presented to illustrate how this interface can be engineered with surface treatments and sizings or coatings to insure thorough wetting, protection of the fiber, chemical bonding between fiber and matrix, toughness and desirable failure modes.
\end{abstract}

